\title{
LAS COMPETENCIAS DE EMPRENDIMIENTO SOCIAL, COEMS: APROXIMACIÓN A TRAVÉS DE PROGRAMAS DE FORMACIÓN UNIVERSITARIA EN IBEROAMÉRICA
}

\author{
POR \\ Nerea SÁENZ BILBAO ${ }^{1}$ y \\ Ana Luisa LÓPEZ VÉLEZ ${ }^{2}$
}

\section{RESUMEN}

El auge que el emprendimiento social tiene en nuestra sociedad cada vez es mayor y muchas universidades han apostado por la educación emprendedora como parte de su misión, adquiriendo un papel de responsabilidad en la sociedad y en el proceso emprendedor, y desarrollando así una "cultura emprendedora". No obstante, se ha constatado la necesidad de establecer un marco común sobre la formación en emprendimiento social, y las competencias que es necesario desarrollar en las personas emprendedoras sociales.

Este artículo tiene como objetivo reflexionar sobre dichas competencias, a través de una revisión de lo planteado en la literatura. A su vez, se pretende comprobar si la propuesta teórica está en consonancia con las competencias que los programas de formación universitaria están desarrollando en esta materia. De esta forma, se contrastó el análisis teórico realizado con los resultados obtenidos de un estudio sobre programas de formación universitarios en emprendimiento social que se realizó dentro del marco de la investigación Alfa-Tuning Latin American Project 2011-2013, financiado por la Comisión Europea. Como parte del estudio, se analizaron las competencias que se están trabajando en dichos programas.

Para ello, se ha hecho un análisis documental de una selección de siete programas de formación de distintas universidades de América Latina y España.

\footnotetext{
${ }^{1}$ Investigadora en formación para el doctorado. Equipo de investigación INNOVA. Facultad de Psicología y Educación. Universidad de Deusto. Dirección de correo electrónico: nerea.saenz@ deusto.es

${ }^{2}$ Investigadora Doctora. Equipo de investigación INNOVA. Facultad de Psicología y Educación. Universidad de Deusto. Dirección de correo electrónico: analuisa.lopez@ deusto.es

REVESCO N 119 - Tercer Cuatrimestre 2015 - ISSN: 1885-8031 - www.ucm.es/info/revesco

http://dx.doi.org/10.5209/rev_REVE.2015.n119.49066

Fecha de recepción: 03/04/2014

Fecha de aceptación: 03/03/2015
} 
Como resultado de la revisión literaria y el análisis de los programas, se delimitó una clasificación de competencias de emprendimiento social, COEMS, que se presenta en este artículo.

Esta clasificación consta de cuatro bloques que pretenden desarrollar las capacidades del alumnado desde una visión de globalidad. Cada bloque de competencias se ilustra con el ejemplo de uno de los programas analizados, debido al trabajo significativo desarrollado en las competencias de dicho bloque. Se hace hincapié, en particular, en aquel bloque de competencias relacionadas con la filosofía y la ética, COEMS 4, como base fundamental para el fomento del compromiso social en el alumnado, marca de identidad de la futura persona emprendedora social.

Palabras Clave: Competencias en emprendimiento social, educación en emprendimiento social, Educación Superior, educación emprendedora, emprendimiento social.

Claves Econlit: I23; L26;L3; M53.

\title{
SOCIAL ENTREPRENEURSHIP COMPETENCES, COEMS: OVERVIEW THROUGH UNIVERSITY EDUCATIONAL PROGRAMS IN LATIN AMERICA AND SPAIN
}

\begin{abstract}
In the last decades, social entrepreneurship is rapidly gaining importance worldwide and many universities have made great efforts to include entrepreneurship education as part of their mission. Thus, higher education institutions are increasingly assuming responsibility towards society, and within its entrepreneurial process, as well as developing an "entrepreneurship culture". However, a social entrepreneurship education common framework is needed, as well as an agreement on the competences to promote in order to develop social entrepreneurs.
\end{abstract}

This article aims to contribute to the reflection upon the competences needed by reviewing and analysing the literature. In addition, the proposal arisen through the literature review is put under consideration while addressing the competences developed by actual university training programs on the topic. Thus, the theoretical analysis is contrasted with the results obtained by a piece of research about university education programs on social entrepreneurship. This study is part of a broader research project, called University Social 
Innovation and Responsibility Model, an Alfa-Tuning Latin American Project 2011-2013, funded by the European Commission. As part of this study, an analysis was carried out on the kind of competences addressed by the educational programs under research. For this purpose, seven training programs from universities in Latin America and Spain were selected and a documentary analysis of each program was carried out.

As a result of the literature review and the documentary analysis of the programs, a classification of social entrepreneurship competences, COEMS, was defined, this is described in this article. The classification consists of four blocks of competences aiming to develop students' capacities globally. As an example of the development of each block of competences, a particular education program described, due to the significance work achieved in relation to those competences. The block of philosophical and ethical competences, COEMS 4, must be particularly underlined, because it is considered fundamental in order to promote learners' social commitment, an essential characteristic of the future social entrepreneurs' identity.

Keywords: Social entrepreneurship competences, social entrepreneurship education, Higher Education, entrepreneurship education, social entrepreneurship.

\section{CONTEXTUALIZACIÓN DEL ESTUDIO}

En un mundo de cambio constante, continua evolución y de incertidumbre económica, es necesario desarrollar una serie de capacidades, herramientas y actitudes para enfrentarse a las exigencias que demanda la nueva sociedad. Para ello, se debe poner el foco en el aprendizaje a lo largo de la vida y la adquisición de competencias emprendedoras, especialmente en el desarrollo de los programas de educación universitaria (European Higher Education Area, EHEA, 2012). Diversas organizaciones internacionales como la UNESCO (2009) hacen una llamada a las instituciones de Educación Superior a asumir la educación emprendedora para responder y anticiparse a las necesidades sociales.

Según distintos autores (El Ebrashi, 2013; Kury, 2012; Moreau y Mertens, 2013; Welsh y Krueger, 2012), la persona emprendedora social quiere lograr una misión social, a través del uso de herramientas de gestión que le permitan dar respuesta a problemas sociales y a retos empresariales. Es un agente de cambio que genera y fomenta valores sociales, y tiene la capacidad de reconocer nuevas oportunidades al servicio de su misión. Para ello, se embarca en un proceso de innovación continua, de adaptación, aprendizaje y acción sin 
sentirse limitado por los recursos existentes. Muestra, a su vez, un alto nivel de responsabilidad hacia la población a la que da servicio y a los resultados logrados. El fin último de su empresa social es la creación de un cambio sostenible en la vida de las personas y en su impacto prima el beneficio de toda la comunidad, por encima de intereses individuales.

Bornstein (2005) describe este tipo de persona como:

(...) un individuo obsesivo que detecta un problema e imagina una nueva solución, que toma la iniciativa para actuar de acuerdo con esa idea, que reúne recursos y construye organizaciones para proteger y comercializar la idea, que surte de energía y de un enfoque sostenido para superar las inevitables resistencias, y que -década tras década - continúa mejorando, fortaleciendo, y engrosando esa idea hasta que lo que en otro momento era una idea marginal se ha convertido en una norma nueva.

(Bornstein, 2005: 22)

Considerando el entorno emprendedor en España, desde distintas fuentes (Arias y Castillo, 2011; GEM, 2012; Wompner, 2012) se recomienda un mayor esfuerzo en conectar la investigación universitaria con los perfiles emprendedores. Para ello, se ve necesario, por un lado, incorporar cada vez más temas de emprendimiento y habilidades relacionadas dentro de los programas de estudio. Pero, a su vez, se requiere que la educación emprendedora impartida permita que los estudiantes desarrollen capacidades con sentido de responsabilidad social, y así participen de manera activa en una sociedad democrática y promuevan transformaciones sociales en pro de la igualdad y la justicia.

A pesar del auge del emprendimiento social en los últimos años, distintas investigaciones han confirmado que hay poca coherencia en el enfoque, sin existir un marco común sobre el concepto de emprender, ni un método referente en relación con la forma de enseñar o educar (Dacin et al. 2011; Fayolle y Gailly, 2008; Osorio y Pereira, 2011; Pfeilstetter, 2011; Sánchez, 2013).

A esta necesidad detectada, se añade el hecho de que cada vez hay más interés por consensuar los conceptos clave que caractericen la formación y docencia en emprendimiento social. Como señalan Ferrer et al. (2014:14) "podemos encontrar puntos en común y un marco compartido cada vez más cercano”. 
Ante este desafío, se considera que las competencias pueden aprenderse y mejorarse (Sánchez, 2013; Taatila, 2010), y se sugiere que la formación debe ser formulada en términos de competencias (Miller, Wesley y Williams, 2012), incluyendo valores éticos, sociales y emocionales. Por ello, en el marco de la educación en emprendimiento social, se ve fundamental el fomento de competencias que promuevan la creatividad, la imaginación, el pensamiento crítico, la colaboración, la proactividad y el trabajo en equipo. De esta manera, se promociona, a su vez, un fuerte sentido de autoestima y confianza (Koiranen y Ruohotie, 2001; Korsgaard, 2011; Pache y Chowdhury, 2012; Raposo y do Paço, 2011; Sánchez, 2013; Villanueva, 2013).

Algunos autores ya reclaman que esta educación emprendedora debe ser compartida a lo largo de toda la institución universitaria, sin distinguirlo como propio únicamente de alguna escuela o facultad en particular. Además apuntan que al menos se necesitan dos cambios para cumplir los objetivos de la educación emprendedora: se deben cambiar los planes de estudio y desarrollar métodos de enseñanza-aprendizaje distintos (Jones, Matlay y Maritz, 2012; Ruskovaara y Pihkala, 2013).

Por este motivo, se centra nuestra atención en un aspecto pedagógico central. El objetivo se basa en analizar las competencias que deben promover los planes y programas de estudio con miras a desarrollar el perfil emprendedor social en el alumnado universitario. De esta forma, se presenta, a continuación, una clasificación de competencias en emprendimiento social, COEMS, realizada a partir de la revisión teórica. Ésta, a su vez, se contrastó con los resultados obtenidos de un estudio sobre programas de formación universitarios en emprendimiento social, y particularmente en relación a las competencias que se están trabajando.

Este estudio es resultado de la investigación realizada en el marco del proyecto del Modelo de Innovación Social Universitaria Responsable (ISUR), Alfa-Tuning Latin America, financiado por la Comisión Europea, liderado por el equipo INNOVA de la Universidad de Deusto y en el que participaron 14 universidades de América Latina, de Argentina, Chile, Colombia, Costa Rica, Ecuador, Guatemala, Perú, Nicaragua, Uruguay y Venezuela; y 2 de Europa, de España y Dinamarca.

Como parte de esta investigación, se realizó un estudio sobre programas de educación en emprendimiento social de distintas universidades (Villa, 2013). Para ello, se dirigió una 
convocatoria a 150 universidades participantes en el proyecto global del Alfa-Tuning Latin American Project 2011-2013. En un primer momento, se recibieron 14 experiencias de programas formativos en emprendimiento social, a las que se les solicitó que registraran los datos fundamentales del programa de formación en una ficha de registro, e incluyeran cualquier documento original que aportara mayor información sobre el programa.

Tras el análisis cualitativo de dichas fichas, y siguiendo los criterios de clasificación de COEMS derivados de la revisión literaria, se realizó una selección en base a las competencias desarrolladas. También se llevó a cabo un análisis de la metodología de enseñanza y aprendizaje, y el modelo de evaluación en que se basaban dichos programas. Finalizado este proceso, 7 casos fueron seleccionados ya que ilustran aspectos relevantes para que los programas formativos universitarios impulsen el emprendimiento social.

A continuación se presenta la clasificación de competencias en emprendimiento social (COEMS). A modo ilustrativo, después de la descripción teórica de cada bloque de competencias, se muestran ejemplos emanados del estudio realizado sobre programas educativos universitarios de América Latina y España. En cada ejemplo, se ilustran y analizan algunas de las competencias de emprendimiento social (COEMS) de un bloque específico.

\section{CLASIFICACIÓN DE COMPETENCIAS DE EMPRENDIMIENTO SOCIAL (COEMS)}

En el contexto actual, las universidades se erigen como un marco pedagógico fundamental donde fomentar las competencias para lograr que los y las futuras profesionales sean personas emprendedoras e implicadas en la mejora de la sociedad. Por ello, las instituciones universitarias han asumido el reto de fomentar la educación emprendedora, convirtiéndose así en un agente destacado dentro de la "sociedad de emprendimiento" (Sánchez y Gutiérrez, 2011).

Según Osorio y Pereira,

(...) es necesario que la universidad, como agente formador y transformador, ayude a los individuos a participar de un proceso de "acción emprendedora", reconociendo sus intenciones, pensamientos, expectativas y creencias personales, así como las limitaciones de su entorno. (Osorio y Pereira, 2011:19) 
Esta formación emprendedora se enfrenta con un reto fundamental que es pasar de la educación "sobre" emprendimiento a una educación "para” el emprendimiento. De esta forma, se deben hacer esfuerzos para que el alumnado adquiera determinadas habilidades y comportamientos que caracterizan a la persona emprendedora (Pache y Chowdhury, 2012).

Ante esto, el fomento de unas determinadas competencias va a ser fundamental en la labor académica. Bisquerra y Pérez (2007:63) conciben la competencia como la "capacidad de movilizar adecuadamente el conjunto de conocimientos, capacidades, habilidades y actitudes necesarias para realizar actividades diversas con un cierto nivel de calidad y eficacia”. Villa y Poblete (2008), por su parte, hacen hincapié en un conjunto de valores que deben formar parte central de las competencias a fomentar.

A su vez, Osorio y Pereira (2011) enfatizan la importancia del desarrollo de unas determinadas competencias en el proceso de enseñanza y aprendizaje para lograr que la persona asuma el desafío de llevar a cabo acciones emprendedoras. No obstante, Taatila (2010) puntualiza que muchos estudios de referencia concuerdan que las competencias realmente emprendedoras se relacionan a menudo con las habilidades psicológicas y sociales, por encima de las habilidades exclusivamente abocadas a crear una empresa. Por ello, Sánchez (2013: 459) aboga por transformar “corazones y mentes” a través del desarrollo de habilidades emocionales.

De esta manera, Martínez y Carmona (2009) definen como competencias emprendedoras (COEM) aquellas que permiten desarrollar un proyecto que genere crecimiento económico y cohesión social, estableciéndose así en un proyecto social integrado.

La particularidad de las COEM, además de favorecer las iniciativas emprendedoras, radica en que ofrece una visión más amplia de las competencias, más allá de lo puramente laboral-empresarial. Contemplan un objetivo más ambicioso que es el de hacer capaces a los sujetos en su dimensión personal (autonomía y autorrealización), social (responsabilidad social y medioambiental) y económica (proyecto empresarial).

(Martínez y Carmona, 2009:84)

Según distintos autores, el objetivo principal de este enfoque de competencias es desarrollar y consolidar la cultura emprendedora. Con este mismo fin, se identifican distintas competencias emprendedoras como relevantes en el perfil emprendedor y las agrupan según 
ámbitos de su quehacer (Arias y Castillo, 2011; Martínez y Carmona, 2009; Moriano, Trejo y Palací, 2001).

Mitchelmore y Rowley (2010, cit. Chell, 2013) plantean un modelo de competencias dividido en cuatro categorías: competencias emprendedoras; competencias de gestión empresarial; competencias para las relaciones humanas; y competencias conceptuales y de relación.

No obstante, en este estudio se ha intentado contribuir presentando una clasificación que no sólo tenga en cuenta aquellas competencias que ayuden a cualquier persona emprendedora a tener éxito. Se pretende ir más allá, poniendo el énfasis en aquellas competencias que la literatura considera claves a la hora de fomentar el desarrollo de una persona emprendedora con un claro compromiso de transformación social. Por ello, fruto de la revisión literaria realizada, se pueden englobar las competencias de emprendimiento social (COEMS) en los siguientes bloques.

Gráfico 1. Clasificación de Competencias de Emprendimiento Social, (COEMS).

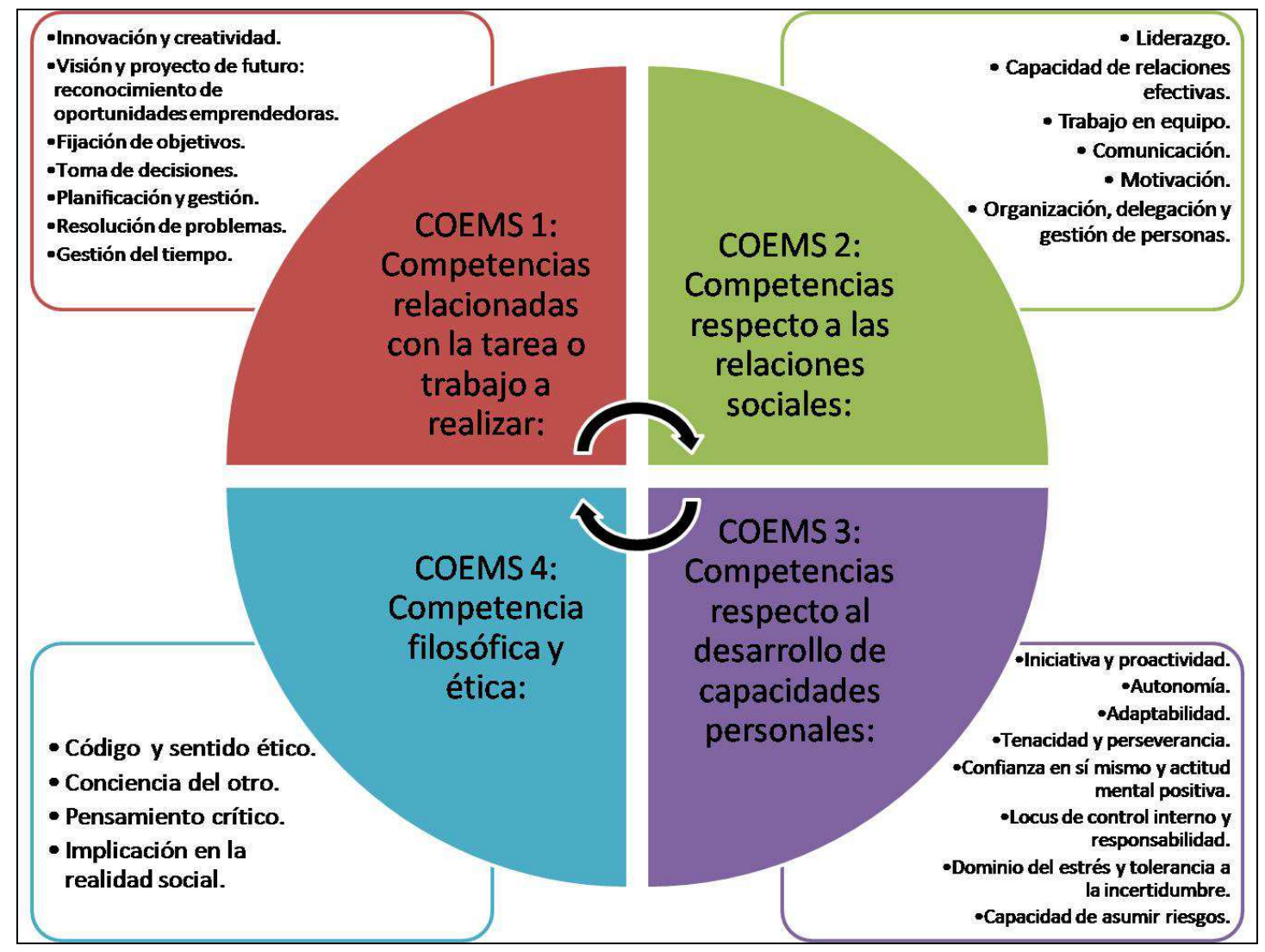

Fuente: Elaboración propia 
Como se puede observar en el Gráfico 1, se presenta una clasificación de las competencias que debieran fomentarse en las personas para desarrollarse como emprendedoras sociales, (COEMS). Se trata de personas que sean capaces de detectar una necesidad social, anticiparse y reconocer las oportunidades emprendedoras, que poseen una idea creativa e innovadora. A su vez, deben tener la capacidad de ponerla en marcha creyendo en sus posibilidades, responsabilizándose e implicando a la sociedad a asumir nuevos retos.

A continuación, se determinan y definen las competencias que conforman cada bloque, y, modo de ilustración, se presentan ejemplos de algunos de los programas analizados, haciendo hincapié en aquellas competencias contempladas. Esto no significa que únicamente se trabaje esta competencia, pero sí su relevancia y el modo en que se pretenden fomentar. Además, las competencias seleccionadas se contextualizan dentro del programa perteneciente con su correspondiente descripción y objetivos.

\section{COEMS 1: COMPETENCIAS RELACIONADAS CON LA TAREA O TRABAJO A REALIZAR}

El primer grupo de competencias están relacionadas con la tarea, con el trabajo a realizar (Chell, 2013; Martínez y Carmona, 2009; Moriano, Trejo y Palací, 2001; Toca, 2010; Villa y Poblete, 2008).

- Innovación y creatividad: Capacidad de dar una respuesta creativa a las demandas y necesidades personales, organizativas, y sociales; modificar tanto los procesos como los resultados; sentir la comodidad y apertura hacia nuevas ideas, enfoques e información.

- Visión y proyecto de futuro: reconocimiento de oportunidades emprendedoras: Capacidad de iniciar, hacer, conseguir y construir una iniciativa; generar ideas e identificar las oportunidades donde otras personas ven caos, contradicción o confusión.

- Fijación continua de objetivos: Capacidad de establecer metas claras desafiantes pero, a la vez, alcanzables.

- Toma de decisiones: Capacidad de elegir la mejor alternativa para actuar, comprometiéndose con la decisión tomada y siendo coherente con la misma.

- Planificación y gestión: Capacidad de organizar de manera ordenada, coherente y pragmática. 
- Resolución de problemas: Capacidad de reconocer los problemas como parte inherente del proyecto y sobrellevarlos con soluciones creativas y actitud positiva.

- Gestión del tiempo: Capacidad de organizar y distribuir correctamente el tiempo de las actividades diarias que realizan para maximizar el resultado, y cumplir lo planificado.

En el análisis de los programas de formación en emprendimiento social se observó que se desarrollan las siguientes COEMS 1: Innovación y creatividad; Visión y proyecto de futuro; y Planificación y gestión.

\section{COEMS 1. INNOVACIÓN Y CREATIVIDAD}

\subsection{Programa Competencias Emprendedoras, de la Universidad Católica de Temuco, Chile}

El programa Competencias emprendedoras, de la Universidad Católica de Temuco (Chile), contempla la Innovación y la Creatividad como una competencia que permita desarrollar en su alumnado el perfil emprendedor. El programa se define como un "curso que pretende fortalecer y desarrollar conductas que favorezcan el perfil emprendedor de los estudiantes". (Villa 2013: 222). Además, responde al plan de desarrollo institucional que promueve una cultura de emprendimiento.

Bajo la Dirección General de Desarrollo Estudiantil, dentro del programa de Formación Humanista y cristiana, es un curso electivo de duración de un semestre con una dedicación de 45 horas para prácticas profesionales en una microempresa. Alrededor de 20 alumnos y alumnas participan de este curso por semestre.

Además de potenciar el perfil de la persona emprendedora, entre los objetivos que plantea este curso está que el alumnado conozca la realidad de su región y de la población emprendedora. A su vez, se pretende sensibilizar al alumnado en la importancia de la microempresa como agente de innovación y desarrollo local.

Con respecto a la COEMS 1 de Innovación y Creatividad, según se plantea en la propia ficha de registro, se considera fundamental a la hora de conseguir,

(...) potenciar una predisposición positiva, habilidades y métodos para el emprendimiento, de manera que al egresar de sus carreras tengan una actitud proactiva para enfrentar el mundo del trabajo, y logren convertirse en una oferta más 
innovadora y efectiva para sus potenciales empleadores o, en su defecto, que desarrollen sus propias iniciativas empresariales. (Ficha de registro, Universidad Católica de Temuco, Chile)

Para lograrlo, las sesiones son participativas. Por un lado, el trabajo individual se centra en que cada estudiante realice presentaciones orales sobre lo aprendido, en su realización de análisis bibliográficos sobre distintos temas. Por otro, se fomenta el trabajo en equipo, a través de los estudios de casos, y la organización de talleres abiertos donde transmitan al resto del grupo lo trabajado.

El programa de Competencias Emprendedoras, además de fomentar la innovación y creatividad, como ejemplo de competencia del bloque COEMS1, se focaliza también en las competencias en relación a los demás (COEMS 2) que desarrollan el trabajo en equipo, y las competencias filosóficas y éticas (COEMS 4), a través del fomento de la competencia código y sentido ético.

\section{COEMS 2: COMPETENCIAS RESPECTO A LAS RELACIONES SOCIALES}

El segundo bloque se centra en las competencias en relación con los demás (Arias y Castillo, 2011; Martínez y Carmona, 2009; McClelland, 1989; Toca, 2010; Villa y Poblete, 2008):

- Liderazgo: Capacidad para dirigir o guiar desde el respeto a los demás; ejercer una influencia sobre las personas; y contribuir a su desarrollo personal y profesional.

- Capacidad de relacionarse de manera efectiva: Capacidad de relacionarse y estar informado entre diferentes colectivos, organizaciones o grupos en diferentes contextos y situaciones, para fortalecerse a través de la generación de redes de contacto y cooperación con otros.

- Trabajo en equipo: Capacidad de integrarse y colaborar de forma activa en la consecución de objetivos comunes con otras personas, áreas y organizaciones.

- Comunicación: Capacidad de comunicarse correctamente y con fluidez con diferentes personas y en diversas situaciones; capacidad de escucha empática y asertiva, siendo tolerante, paciente, receptivo, mostrando interés, no descalificando; interpretar las emociones; y aportar la retroalimentación adecuada a cada persona. 
- Motivación: Capacidad de animarse y animar a las personas con quien se trabaja, tanto en el presente como en el futuro, para desarrollar con interés su trabajo.

- Organización, delegación y gestión de personas: Capacidad de escoger adecuadamente a las personas que van a formar parte de su proyecto; coordinar el equipo; y repartir tareas y funciones de forma competente.

En el estudio de los programas de formación iberoamericanos, se observó que se fomentan las siguientes COEMS 2: Liderazgo; Capacidad de relaciones efectivas; Trabajo en equipo; Comunicación; Organización, delegación y gestión de personas.

\section{COEMS 2: TRABAJO EN EQUIPO}

\subsection{Modelo de Formación de Emprendedores Sociales basado en Competencias, de la Universidad de Deusto, Bilbao, España}

La Universidad de Deusto, desde el campus de Bilbao, España, ha desarrollado el Modelo de Formación de Emprendedores Sociales basado en Competencias. Se trata de un programa formativo en emprendimiento diseñado en base a competencias genéricas, seleccionadas como claves para el desarrollo del emprendimiento social y "orientadas a transformar la idea emprendedora en una propuesta de valor" (Ficha de registro, Universidad de Deusto, España). Además, desarrolla las competencias específicas que deben cubrir las principales áreas de una iniciativa empresarial.

El programa se implementa desde la Facultad de Psicología y Educación y pretende

(...) contribuir al desarrollo de una cultura de iniciativa y emprendimiento en la sociedad, a lo largo de la elaboración de proyectos o iniciativas sociales durante el proceso formativo y realizar una contribución significativa en la generación de un perfil profesional que se integre y promueva la generación de nuevas iniciativas empresariales de carácter social. (Ficha de registro, Universidad de Deusto, España).

Se trata de un curso voluntario, de 150 horas de duración, o 6 créditos ECTS (European Credit Transfer System) y dirigido en torno a 25 profesionales de ONG e instituciones del tercer sector. No obstante, se plantea como una modalidad susceptible de ser incorporada a distintos programas de educación universitaria. 
En cuanto a los objetivos, se espera que los participantes a lo largo de la formación identifiquen los valores, objetivos y competencias necesarias para ser una persona emprendedora social, así como el desarrollo de competencias genéricas y específicas. Respecto a la creación de empresas sociales, el objetivo es diferenciar los aspectos concretos de una empresa social, y conocer los principios éticos y de justicia social en los que se basa una empresa de estas características. Además, el programa persigue desarrollar cauces de colaboración entre la Universidad y el entorno, como las instituciones locales, regionales u ONG, para la transferencia de los aprendizajes construidos.

Uno de los aspectos que caracterizan este programa es la iniciativa o proyecto social que los alumnos y alumnas diseñan, a lo largo de la formación, en grupos. Por esto, la competencia trabajo en equipo (COEMS 2) se considera fundamental.

Para la creación de un proyecto o iniciativa social, se utiliza una metodología activa, que consta de sesiones presenciales y de la realización de un estudio de caso. Este estudio de caso, que se centra en el análisis de la realidad y la propuesta de un proyecto social concreto, se va desarrollando a lo largo de todo el curso, donde se van solucionando las cuestiones que surgen respecto a la competencia que se esté trabajando en ese momento mediante diferentes técnicas de grupo. A su vez, cada equipo cuenta con un tutor que ofrece apoyo, asesoramiento y seguimiento. De este modo, se desarrollan competencias como la Comunicación; Liderazgo; y Organización, delegación y gestión de personas (COEMS 2).

Este programa también hace hincapié en competencias relacionadas con la tarea o trabajo a realizar (COEMS 1), con el fin de convertir esa idea emprendedora en un plan de negocio. Por ello, entre las COEMS 1, se desarrollan la Innovación, Visión y proyecto de futuro: reconocimiento de oportunidades emprendedoras y Planificación y gestión. Por último, también se resalta la importancia de la competencia que fomente un código y sentido ético (COEMS 4).

\section{COEMS 3: COMPETENCIAS RESPECTO AL DESARROLLO DE CAPACIDADES PERSONALES}

En el tercer bloque de COEMS se hace alusión a aquellas competencias transversales que promuevan el desarrollo de habilidades y capacidades personales, entre ellas se encuentran (Martínez y Carmona, 2009; Moriano, Trejo y Palací, 2001; Toca, 2010): 
- Iniciativa y proactividad: Capacidad de promover iniciativas; introducir nuevos productos, servicios o tecnología innovadores; y anticiparse a los problemas futuros, necesidades o cambios.

- Autonomía: Capacidad de búsqueda de independencia y libertad de acción.

- Adaptabilidad: Flexibilidad para adoptar los cambios.

- Tenacidad y perseverancia: Capacidad de constancia en una tarea o acción de principio a fin; llevarla a cabo con sacrificio, empeño y determinación; superar las primeras adversidades o dificultades; y sobreponerse al fracaso.

- Confianza en sí mismo y actitud mental positiva: Capacidad de valorarse a uno/a mismo/a, capacidades y habilidades para desarrollar con éxito una iniciativa o proyecto.

- Locus de control interno y responsabilidad: Percibir que el éxito depende de uno/a mismo/a, más que de circunstancias externas; asumir el protagonismo de sus propios actos.

- Dominio del estrés y tolerancia a la incertidumbre: Capacidad de control en situaciones difíciles o agobiantes; y soportar las tensiones y la inseguridad.

- Capacidad de asumir riesgos: Capacidad de aceptar los riesgos y asumir las responsabilidades que esto supone.

En el estudio realizado sobre la educación superior en emprendimiento social, se destacaron las siguientes COEMS 3: Locus de control interno y responsabilidad; Dominio del estrés y tolerancia a la incertidumbre; y Capacidad de asumir riesgos.

\section{COEMS 3: CAPACIDAD DE ASUMIR RIESGOS}

\subsection{Programa Emprendimiento Social, Pontificia Universidad Javeriana de Cali, Colombia}

El Programa de Emprendimiento Social, de la Pontificia Universidad Javeriana de Cali, en Colombia, se describe como,

(...) un curso que motiva al estudiante a desarrollar su potencial creativo, a través del acompañamiento y el levantamiento de su proyecto de vida a la luz de la experiencia de vida que pueda encontrar en el trabajo de campo con los líderes de organizaciones, fundaciones y proyectos sociales de su región. En él, se aprende sobre cómo existen 
una serie de organizaciones que se comprometen con el desarrollo de proyectos sociales autosostenibles. (Villa 2013: 238)

Pretenden, además, tener un mejor conocimiento acerca de lo que hacen este tipo de organizaciones a nivel social en el país, buscando contribuir en cómo cambiar el mundo.

El Programa se lidera a través del Área de Emprendimiento del Departamento de Gestión de Organizaciones, perteneciente a la Facultad de Ciencias Económicas y Administrativas. Se trata de un curso electivo, accesible a estudiantes de cualquier programa académico y de 48 horas presenciales de duración.

El Programa tiene por objetivo:

(...) sensibilizar al estudiante sobre la importancia del emprendimiento social y su impacto en el desarrollo de una región, además de buscar orientarle a escoger la opción de ser un líder con espíritu solidario con capacidad de evaluar las necesidades de su región para que su opción llegue a ser la entrega a una comunidad en servicio como vía de desarrollo personal y profesional.

(Ficha de registro, Pontificia Universidad Javeriana de Cali, Colombia)

En cuanto a las competencias respecto al desarrollo de capacidades personales (COEMS 3), el programa fomenta la capacidad de asumir riesgos, formando "individuos en la sociedad civil protagonistas en la mejora de las condiciones sociales, con el potencial necesario para promover un proyecto social de alto impacto". (Ficha de registro, Pontificia Universidad Javeriana de Cali, Colombia). Se enfatiza además, la competencia tolerancia a la incertidumbre, fomentando el emprendimiento social ante la magnitud de diversos problemas sociales y ante cualquier situación económica o social.

Para ello, se utiliza una metodología fundamentada en el aprendizaje significativo "que busca activar la autonomía y corresponsabilidad en el estudiante, al hacerle responsable, no solo por lo que aprende sino por lo que aprenden sus compañeros de equipo" (Ficha de registro, Pontificia Universidad Javeriana de Cali, Colombia). Se trabaja con tres estrategias de enseñanza-aprendizaje: aprendizaje colaborativo, aprendizaje basado en proyectos y mapas conceptuales. 
El Programa Emprendimiento Social, además de fomentar las competencias capacidad de asumir riesgos y tolerancia a la incertidumbre (COEMS 3), también promueven la visión y proyecto de futuro (COEMS 1); la comunicación y capacidad de relaciones efectivas (COEMS 2); y el pensamiento crítico (COEMS 4).

\section{COEMS 4: COMPETENCIA FILOSÓFICA Y ÉTICA}

El componente ético tiene una importancia incuestionable en el trabajo de la persona emprendedora social, ya que va a contribuir al cambio de patrones de comportamiento, y de organización, entre otros. Es la marca de identidad que caracteriza a una persona emprendedora como social.

Esto también es un aspecto que se ha observado en distintas investigaciones. En un estudio realizado por Arthur, Hisrich y Cabrera (2012) uno de los factores de éxito destacados por las personas emprendedoras entrevistadas era que sus operaciones de negocios se realizaban dentro de unos valores éticos y de transparencia. A su vez, Miller et al. (2012) al encuestar a 150 emprendedores y emprendedoras sociales en activo, pudieron constatar que en su totalidad daban valores especialmente altas a las competencias relacionadas con el sentido ético y moral. Sin embargo, no era un tema que tuviera un peso importante en los cursos sobre emprendimiento social, únicamente en un $39 \%$ de los cursos se contemplaban. Para dichos autores, las competencias filosóficas y de sentido ético se manifestaron como fundamentales a la hora de que las personas emprendedoras sociales en activo no pierdan de vista su misión, y se centren en el valor económico más que en el social.

José Antonio Marina (2010) defiende la necesidad de reconocer la importancia de la competencia filosófica y ética, que permita definir qué proyectos se consideran más valiosos para la sociedad. Según él, no interesa educar la competencia de emprender si no se establecen unos objetivos personales y sociales, concretados en un conjunto de valores compartidos. Considera que uno de los fallos del actual sistema de competencias es que elude la educación ética y la reflexión crítica.

Por ello, como cuarto bloque que caracteriza el aspecto social de las competencias a las que se hace alusión en esta investigación, están las relacionadas con la competencia filosófica y ética (Martínez y Carmona, 2009; Toca, 2010; Villa y Poblete, 2008): 
- Código y sentido ético: Capacidad de acción y comportamiento regido por normas morales basadas en el respeto a las personas y a la naturaleza.

- Conciencia del otro: Capacidad de analizar, entender y actuar en función de las problemáticas y necesidades de las personas; y tener en cuenta los distintos aspectos, tanto profesionales como personales, que influyen en cada persona.

- Pensamiento crítico: Capacidad de interrogarse por diferentes cosas, y mantener interés por los fundamentos en los que se asientan las ideas, las acciones, las valoraciones y juicios tanto propios como ajenos.

- Implicación en la realidad social: Capacidad de comprometerse e inferir en aspectos sociales y culturales a través de la convivencia social en diferentes realidades.

En lo que se refiere a los programas de formación en emprendimiento social analizados en el estudio, se ve un especial énfasis en el desarrollo de todas las COEMS 4: Código y sentido ético; Conciencia del otro; Pensamiento crítico; e Implicación en la realidad social.

\section{COEMS 4: IMPLICACIÓN EN LA REALIDAD SOCIAL}

\subsection{Emprededorismo Social, Universidad de Paraná, Brasil.}

La Pontificia Universidad Católica de Paraná, en Brasil, cuenta con el programa formativo Emprendedorismo Social dentro de su Escuela de Negocios. Como parte de la oferta de estudios de postgrado, es un curso optativo de un semestre de duración. Con respecto al modo en que se pretende fomentar la COEMS 4 de implicación con la realidad social, cabe destacar las 30 horas de prácticas profesionales que se realizan en cooperativas de la comunidad.

Según lo define la propia universidad, este programa se describe como

(...) una intervención de los estudiantes en comunidades necesitadas, en la región metropolitana de Curitiba situada en el Área de Protección Ambiental de Guaratuba donde viven 150 familias, y 24 de ellas están organizadas en una cooperativa de productores de plátanos. (Villa 2013:238)

Entre los objetivos del programa para el desarrollo de las competencias relacionadas con la filosofía y la ética (COEMS 4), se hace un gran énfasis en que el alumnado 
experimente las necesidades de distintos colectivos en situación de vulnerabilidad a través de su implicación con organizaciones de la comunidad. De esta manera, se familiariza con la realidad y el contexto social y cultural en que viven dichas personas.

Esto se pretende conseguir a través de metodologías participativas, como tertulias, visitas de campo, y, en particular, con el desarrollo de proyectos en colaboración con las organizaciones comunitarias. Al salir a la realidad mediante la práctica profesional, el alumnado suele experimentar un "choque cultural" con realidades desconocidas hasta ese momento. Además, se organizan equipos de trabajos diversos, donde se da un intercambio activo con profesionales de distintas disciplinas, al igual que con agentes comunitarios.

De esta manera, además de fomentar la implicación en la realidad social, dentro de las competencias relacionadas con la filosofía y ética (COEMS 4), también se promueve la conciencia del otro. Con respecto a los otros bloques de competencias, el programa tiene como objetivo desarrollar competencias respecto a las relaciones sociales (COEMS 2), de liderazgo y trabajo en equipo; y competencias respecto a las capacidades personales (COEMS 3), particularmente, relativas al locus de control interno y la responsabilidad. No obstante, para que la formación de dicho alumnado fuera más completa, en este programa se observa que no se han considerado competencias más abocadas a la tarea o al trabajo (COEMS 1).

\section{REFLEXIONES FINALES}

El compromiso por la responsabilidad social y la innovación ha hecho que muchas universidades e instituciones de Educación Superior asuman transformaciones en los cuatro pilares fundamentales de su quehacer, en el ámbito pedagógico, epistemológico, de acción social y de gestión. Particularmente en el ámbito educativo, se han contemplado avances en el curriculum y los programas formativos, tanto en los niveles de grado como de pregrado. Aunque en la mayoría de los casos, estos cambios están relacionados con determinados departamentos y facultades, cada vez hay una mayor conciencia de que para fomentar profesionales comprometidos con la realidad social, los cambios pedagógicos deben ser transversales a la institución educativa, en su globalidad.

Y esto es fundamental a la hora de intentar fomentar una actitud de emprendimiento social, no sólo en aquel alumnado interesado en dedicarse al mundo de la empresa, sino a cualquier profesional. La capacidad de emprender, de innovar, de mejora y aprendizaje 
continuo y de implicación en la justicia social y la transformación social sistémica debe ser una marca de identidad de nuestras futuras generaciones.

Para ello, es necesario que las instituciones de formación eduquen "para" el emprendimiento, e inculquen unos valores éticos y sociales que impregnen las capacidades, habilidades, actitudes y comportamientos de su alumnado. No obstante, aunque cada programa educativo pretende responder a esta necesidad, se percibe la prioridad de establecer un consenso y un marco común que siente las bases de las competencias a desarrollar, la metodología de enseñanza y aprendizaje adecuada, y el sistema de evaluación que permita evaluar los avances desarrollados por cada alumno y alumna.

Según lo considerado en la literatura, cuatro son los bloques donde se deben fomentar competencias que permitan desarrollar al alumnado en su globalidad, para lograr fomentar un perfil emprendedor, con una clara impronta social, COEMS. Estos cuatro bloques responden a: competencias relacionadas con la tarea o el trabajo a realizar (COEMS 1); a construir relaciones con los demás (COEMS 2); a desarrollar las capacidades personales e individuales (COEMS 3); y a fomentar la faceta filosófica y ética (COEMS 4).

Estas competencias persiguen desarrollar un perfil de una persona emprendedora social, cuya motivación principal sea generar un cambio social profundo y duradero y no el beneficio económico; y cuyo objetivo final sea la justicia social. Esta motivación por emprender para ayudar a resolver un problema o necesidad social y la creación de valor sostenible son las características clave que diferencian el perfil de una persona emprendedora social frente al perfil de cualquier persona emprendedora.

En particular, se ve relevante que los programas educativos universitarios pongan el énfasis en las COEMS 4, relativas a los valores éticos, si se pretende lograr personas emprendedoras que estén comprometidas con las transformaciones y cambios en pro de la justicia social. Se observa que muchos programas ponen el énfasis en las competencias que desarrollan el emprendimiento, no obstante, en aquellos programas donde se hace hincapié en las COEMS 4, es donde se hace explícito y evidente el desarrollo de la dimensión social en el alumnado.

Prueba de que esto está cambiando son los cuatro programas analizados presentados en este artículo. El análisis se ha centrado en el modo que cada programa pretende desarrollar una competencia de cada bloque en concreto: COEMS 1, Innovación y creatividad; COEMS 
2, Trabajo en equipo; COEMS 3, Capacidad de asumir riesgos y COEMS 4, Implicación con la realidad social. No obstante, es de destacar que cada uno de los programas formativos tiene como objetivo fomentar competencias de más de un bloque. Se observa, por ello, que dichos programas pretenden desarrollar al individuo en su globalidad, y particularmente relevante es el hecho de que en su mayoría se pone el énfasis en las competencias relativas a la filosofía y la ética, COEMS 4, como eje conductor de la experiencia de enseñanza y aprendizaje.

No obstante, se debe destacar que en muchos de los programas estudiados no se hace hincapié específico al desarrollo de las capacidades personales, COEMS 3. Esto puede ser por la falsa creencia de que estas capacidades son innatas o que ya se han desarrollado anteriormente en la educación reglada. Según lo que apunta la literatura, el fomento de las capacidades personales es fundamental a la hora de que la formación en emprendimiento social considere al alumnado como una persona en su globalidad, y como tal, se le proporcionen las herramientas que le permitan desarrollarse como persona completa.

Según lo analizado, tanto en la teoría como en los programas estudiados, hay una gran variedad entre las competencias a desarrollar y el modo en que llevarlo a cabo. Se aprecia una necesidad de llegar a consensuar y establecer unos marcos comunes en las competencias que son fundamentales desarrollar en cualquier formación en emprendimiento social. Este estudio puede sentar las bases de los bloques de COEMS que deben ser contemplados en cualquier programa educativo.

Además, la investigación que aquí se ha presentado, permite abrir nuevos ámbitos de análisis en relación a las COEMS. Se ve la necesidad de profundizar en el abanico de competencias que forman parte de cada bloque, y definirlas de una forma más precisa. Incluso es relevante poner el énfasis en competencias que no han emanado en este estudio pero que están latentes en los programas de formación analizados, aunque no de manera explícita. Se observa que existe un conjunto de competencias específicas referidas a la gestión empresarial que debieran formar parte de las COEMS, al igual que competencias relativas a la sostenibilidad y al medioambiente.

Pero a su vez, se percibe la relevancia de seguir investigando y profundizando en este marco. Por un lado, para conocer si el fomento de las COEMS consideradas realmente logra desarrollar un perfil de alumnado egresado que sea emprendedor y esté comprometido con la justicia social. Y por otro, para determinar cuáles son las metodologías de enseñanza y 
aprendizaje, y los modelos de evaluación que puedan fomentar dichas competencias y evaluarlas. El proceso educativo no se puede evaluar de una forma precisa si no se han determinado de forma concreta las competencias que el/la futuro/a profesional del emprendimiento social debe adquirir.

El fomento de la investigación en estos ámbitos que alimente el debate y permita llegar a acuerdos relativos a un marco consensuado referente a la formación en emprendimiento social, permitirá dar pasos de mejora y de innovación educativas que fortalezca a las instituciones de formación, particularmente en educación superior. Y, en consecuencia, ayudará a dar pasos para lograr futuras personas y profesionales de distintos ámbitos implicados en la transformación social sostenible que lleve a nuevos modelos de convivencia y sociedades más justas.

\section{BIBLIOGRAFÍA}

ARIAS, C.M. Y CASTILLO, E. (2011) La educación para el emprendimiento y empresarismo virtual: potencialidades. Revista Virtual Universidad Católica Del Norte [en línea], (Febrero-Mayo). Disponible en:

http://estudiosterritoriales.org/articulo.oa?id=194218638001 ISSN: 0124-5821.

ARTHUR, S.J., HISRICH, R.D. Y CABRERA, A. (2012) The Importance of Education in the Entrepreneurial Process: A World View. Journal of Small Business and Enterprise Development, vol. 19, nº 3, pp. 500-514.

BISQUERRA, R. Y PÉREZ, N. (2007) Las competencias emocionales. Educación XX1, vol. 10, pp. 61-82.

BORNSTEIN, D. (2005) Cómo Cambiar el Mundo. Los Emprendedores Sociales y el Poder de las Nuevas Ideas. Barcelona: Debate.

CHELL, E. (2013) Review of skill and the entrepreneurial process. International Journal of Entrepreneurial Behaviour \& Research, vol. 19, n 1, pp. 6-31.

DACIN, T.; DACIN, P.A. Y TRACEY, P. (2011) Social entrepreneurship: a critique and future directions. Organization Science, vol. 22, nº 5, pp. 1203-1213.

EL EBRASHI, R. (2013). Social entrepreneurship theory and sustainable social impact. Social Responsibility Journal, vol. 9, nº 2, pp. 188-209.

EUROPEAN HIGHER EDUCATION AREA, EHEA (2012) Making the Most of Our Potential: Consolidating the European Higher Education Area. Bucharest Communiqué. Final Version. Disponible en : 


\section{http://www.ehea.info/Uploads/(1)/Bucharest\%20Communique\%202012(2).pdf}

FAYOLLE, A. Y GAILLY, B. (2008) From craft to science. Teaching models and learning processes in entrepreneurship education. Journal of European Industrial Training, vol. 32, $\mathrm{n}^{\circ} 7$, pp. 569-593.

FERRER-CERVERÓ, V., CABRERA-SANTACANA, O. E., ALEGRE-BENERIA, R. M., MONTANÉ-LOPEZ, A., SÁNCHEZ-VALVERDE-VISUS, C., Y ALAIZ-CHUECA, E. (2014) El perfil del emprendedor social del estudiantado de los Grados de Educación Social, Pedagogía y Trabajo Social en la Universidad de Barcelona. [En línea] REIRE, Revista d'Innovació i Recerca en Educació, vol. 7, $\mathrm{n}^{\mathrm{o}}$ 1. Disponible en: http://www.ub.edu/ice/reire.htm. ISSN: 2013-2255.

GLOBAL ENTREPRENEURSHIP MONITOR (GEM) (2012) Informe GEM España 2012.

Disponible en: http://www.gem-spain.com.

JONES, C.; MATLAY, H. Y MARITZ, A. (2012) Enterprise education: for all, or just some? Education +Training, vol. 54, $\mathrm{n}^{\circ}$ 8/9, pp. 813-824.

KORAINEN, M. Y RUOHOTIE, P. (2001. In the Pursuit of Conative Constructs into Entrepreneurship Education. Journal of Entrepreneurship Education, vol. 3, pp. 9-22.

KORSGAARD, S. (2011) Opportunity formation in social entrepreneurship. Journal of Enterprising Communities: People and places in the global economy, vol. 5, $\mathrm{n}^{\circ}$ 4, pp. 265 285.

KURY, K.M. (2012) Sustainability meets social entrepreneurship: a path to social change through institutional entrepreneurship. International Journal of Business Insights and Transformation (IJBIT), vol. 4, $\mathrm{n}^{\circ}$ 3, pp. 67- 71 .

MARINA, J.A. (2010) La competencia de emprender. Revista de Educación, 351, pp. 49-71.

MARTÍNEZ, F. Y CARMONA, G. (2009) Aproximación al concepto de "competencias emprendedoras": Valor social e implicaciones educativas. REICE. Revista Iberoamericana Sobre Calidad, Eficacia y Cambio en Educación, vol. 7, n 3, pp. 82-98.

MCCLELLAND, D.C. (1989) Estudio de la motivación humana. Madrid: Narcea. ISBN: 8427708610 .

MILLER, T.L.; WESLEY II, C.L. Y WILLIAMS, D.E. (2012) Educating the minds of caring hearts: comparing the views of practitioners and educators on the importance of social entrepreneurship competencies. Academy of Management Learning \& Education, vol. 11, no 3, pp. 349-370.

MOREAU, C. Y MERTENS, S. (2013) Managers' competences in social enterprises: which specificities? Social Enterprise Journal, vol. 9, nº 2, pp. 164-183. 
MORIANO, J.A.; TREJO, E. Y PALACÍ, F.J. (2001) El perfil psicosocial del emprendedor: un estudio desde la perspectiva de los valores. Revista de Psicología Social, vol. 16, $\mathrm{n}^{\circ} 2$, pp. 229-242.

OSORIO, F.F. Y PEREIRA, F. (2011) Hacia un modelo de educación para el emprendimiento: una mirada desde la teoría social cognitiva. Cuadernos de Administración, vol. 24, $\mathrm{n}^{\mathrm{o}}$ 43, pp. 13-33.

PACHE, A.C. Y CHOWDHURY, I. (2012) Social entrepreneurs as institutionally embedded entrepreneurs: Toward a new model of social entrepreneurship education. Academy of Management Learning \& Education, vol. 11, n 3, pp. 494-510.

PFEILSTETTER, R. (2011) El emprendedor. Una reflexión crítica sobre usos y significados actuales de un concepto. Gazeta de Antropología, 27 (1). Disponible en: http://hdl.handle.net/10481/15684. ISSN: 0214-7564.

RAPOSO, M. Y DO PAÇO, A. (2011) Entrepreneurship education: Relationship between education and entrepreneurial activity. Psicothema, vol. 23, n 3, pp.453-457.

RUSKOVAARA, E. Y PIHKALA, T. (2013) Teachers Implementing Entrepreneurship Education-Classroom Practices. Education + Training, vol. 55, nº 2, pp. 204 -216.

SÁNCHEZ, J.C. Y GUTIÉRREZ, A. (2011) Entrepreneurship research in Spain: developments and distinctiveness. Psicothema 2011, vol. 23, no 3, pp.458-463.

SÁNCHEZ, J.C. (2013) The impact of an Entrepreneurship Education Program on entrepreneurial competencies and Intention. Journal of Small Business Management, vol. $51, \mathrm{n}^{\mathrm{o}} 3$, pp. 447-465.

TAATILA, V.P. (2010) Learning entrepreneurship in higher education. Education + Training, vol. 52, $\mathrm{n}^{\mathrm{o}}$ 1, pp. 48-61.

TOCA, C. (2010) Consideraciones para la formación en emprendimiento: explorando nuevos ámbitos y posibilidades. Estudios Gerenciales, vol. 26, nº 17, pp. 41-60.

UNESCO (2009). 2009 World Conference on Higher Education: The New Dynamics of Higher Education and Research for Societal Change and Development. Communiqué Declaration. Paris, 5 - 8 July 2009. Disponible en : http://unesdoc.unesco.org/images/0018/001832/183277e.pdf

VILLA, A. (ed.) (2013) Un Modelo de Evaluación de Innovación Social Universitaria Responsable (ISUR). Tuning - América Latina. Bilbao: Publicaciones de la Universidad de Deusto. 
VILlA, A. Y POBLETE, M. (2008) Aprendizaje Basado En Competencias: Una Propuesta Para La Evaluación De Las Competencias Genéricas. Bilbao: Mensajero. ISBN: 978-84271-2833-0.

VILLANUEVA, S.D. (2013) Las competencias dentro del rol profesional: diferencias entre la Educación Superior (universitaria) y las demandas del mercado laboral. Revista Debate Universitario, [en línea] 1, (2m) pp. 44-65. Buenos Aires, CAEE-UAI. Disponible en: http://ppct.caicyt.gov.ar/index.php/debate-universitario/article/viewFile/1605/pdf. ISSN: 2314-1530.

WELSH, D.H.B. Y KRUEGER, N. (2012) The evolution of social entrepreneurship: what have we learned? Journal of Technology Management in China, vol. 7, $\mathrm{n}^{\mathrm{o}}$ 3, pp. 270-290.

WOMPNER, F.H. (2012) El emprendimiento como factor de movilización social. Nómadas. Revista Crítica de Ciencias Sociales y Jurídicas, vol. 36, $\mathrm{n}^{\circ}$ 4. Disponible en: http://www.redalyc.org/articulo.oa?id=18126450015 